\title{
Modeling Aerodynamic Discontinuities and the Onset of Chaos in Flight Dynamical Systems
}

M. Tobak,

G. T. Chapman, Ames Research Center, Moffett Field, California

A. Ünal, University of Santa Clara, Santa Clara, California

\section{N/Sก}

National Aeronautics and

Space Administration

Ames Research Center

Moffett Field, California 94035 


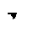

, 
Modeling aerodynamic discontinuities and the onset of chaos in flight dynamical systems
M. TOBAK*
G. T. CHAPMAN*
A. UNAL**

\begin{abstract}
Various representations of the aerodynamic contribution to the aircraft's equations of motion are shown to be compatible within the common assumption of their Fréchet differentiability. Three forms of invalidating Fréchet differentiability are identified, and the mathematical model is amended to accommodate their occurrence. Some of the ways in which chaotic behavior may emerge are discussed, first at the level of the aerodynamic contribution to the equations of motion, and then at the level of the equations of motion themselves.
\end{abstract}

Key words: Unsteady aerodynamics, Nonlinear flight dynamics, Aerodynamics, Nonlinear dynamical systems.

MODÉLISATION DES DISCONTINUITÉS AÉRODYNAMIQUES ET L'INITIATION DU CHAOS AUX SYSTEMES DYNAMIQUES DU VOL

*NASA Ames Research Center, Moffett Field, California 94035 USA. * University of Santa Clara, Santa Clara, California 95053 USA. 
Modeling aerodynamic discontinuities and the onset of chaos in flight dynamical systems

Analyse

On démontre que plusieurs représentations de la contribution aerodynamique aux équations du mouvement de l'avion se trouvent compatibles sous l'hypothèse commune de leur differentiabilité de Fréchet. On identifie trois formes d'invalidation de la differentiabilité de Fréchet, et on amend le modèle mathématique pour accommoder l'événement. Les voies diverses sur lesquelles des proprietés chaotiques peuvent s'élèver sont discutées d'abord au niveau de la contribution aérodynamique aux équations du mouvement, et ensuite au niveau des équations du mouvement elles-mèmes.

Mots clés: Aérodynamique non-stationnaire, Dynamique du vol nonlineaire, Aérodynamique, Systèmes dynamiques nonlineaires 


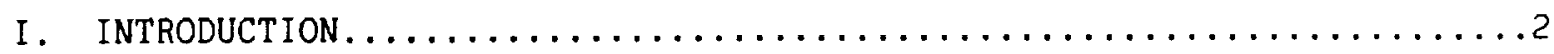

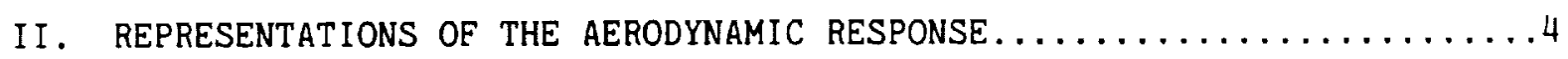

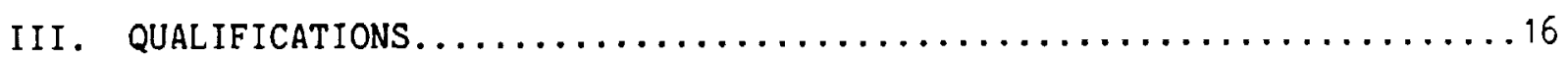

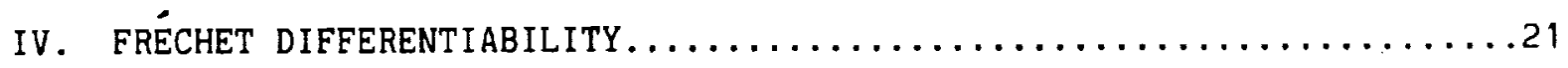

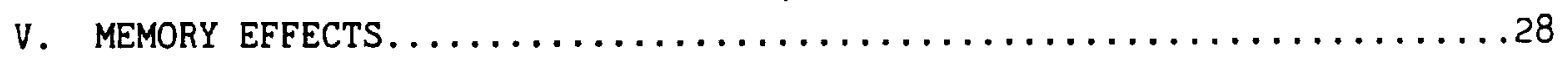

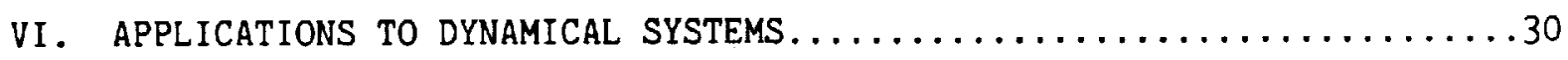

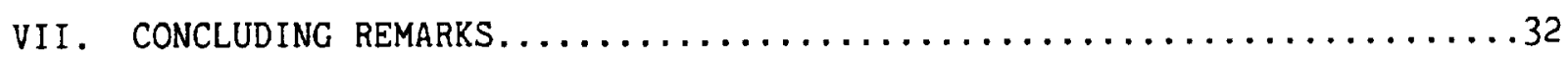

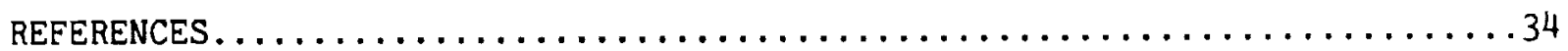


Flight dynamics is the field of research concerned with the dynamical behavior of vehicles in flight. Studies in this field began with Bryan's "Stability in Aviation" [1] at the very beginning of heavier-than-air flight itself. Reporting on progress 25 years later, B. Melville Jones [2] defined the subject's principal task as follows:

Given the shape of the aeroplane and the properties of the air through which it moves, the air reactions $X, Y, Z, L, M, N$ depend on the motion of the aeroplane relative to the air; that is to say upon the six variables $U, V, W, P, Q, R$ and their rates of change with respect to time. In practice, the principal difficulty lies in determining the relationships between $X, Y, \ldots$ and $U, V, \ldots$ The establishment of these relationships with sufficient realism is what we now recognize as the province of mathematical modeling. Forced by the continual expansion of the aircraft's performance envelope, our own studies in the mathematical modeling of the aerodynamic characteristics in flight dynamics have undergone a continual deepening in the effort to maintain sufficient realism over the intervening years [3-7].

Since the $1960 \mathrm{~s}$, our aim has been to provide a consistent formulation and theoretical method for studying the nonlinear aspects of problems in flight dynamics. In recent years, we have become increasingly aware that our studies could be enhanced by linking them to the common features of parallel studies in allied fields. The unifying medium is the rapidly growing body of theory underlying research in nonlinear dynamical systems. We found, first, that our approach to modeling the aerodynamic contribution to the aircraft's equations of motion, involving nonlinear indicial responses and generalized 
superposition integrals [8], could be made compatible with one originating in the field of electrical circuits and systems, based on nonlinear algebraic functional expansions [9]. The reformulation in terms of algebraic functional expansions, which have the virtue of concreteness, clarified some of the essential properties of the indicial response. Second, recognizing the natural connection between our formulation of the indicial response and ideas from bifurcation theory led us in turn to emphasize the central role played by Fréchet differentiability in the formulation. In [10], we proposed that a theory for enumerating the means of invalidating Eréchet differentiability of the response offered an alternative to bifurcation theory with potentially greater scope, and in [11] we began such a theory with an account of the means that we had currently identified. Finally, in [11] we recognized that a potentially important issue emerges when we try to implement the accommodation within the mathematical model of bifurcation phenomena involving timedependent equilibrium states. We saw that memory effects become more important with each successive bifurcation, implying that a chaotic equilibrium state would require information about the complete past history of the motion to ensure a complete specification of the indicial response.

The occasion of this conference, designed to encourage interactions between researchers in a variety of disciplines, gives us an opportunity to present our most recent findings in a more general setting than before. First, we shall try to demonstrate the compatibility that exists between various representations of the aerodynamic response, within the common assumption of their Fréchet differentiability. Then, we shall enumerate with physical examples the means of invalidating Fréchet differentiability that we have Identified. This will lead to a discussion of some of the ways in which chaotic behavior may emerge, first at the level of the aerodynamic 
contribution to the equations of motion, and then at the level of the equations of motion themselves.

II. REPRESENTATIONS OF THE AERODYNAMIC RESPONSE

We intend to demonstrate that various representations of the aerodynamic force and moment response to arbitrary maneuvers are compatible, given the common assumption of their Fréchet differentlability. However, for clarity, we limit attention to the response in lift coefficient $C_{L}$ to a maneuver involving only a single degree of freedom, namely, an arbitrary variation of angle of attack a with time $\xi$.

Figure 1 illustrates the motion under study when it is referred to an $X, Y$ coordinate system that is fixed in space. The aircraft is assumed to have started impulsively from rest in the distant past $\xi \rightarrow-\infty$ with fixed axial velocity $U_{0}$ and zero vertical velocity. It passes through the origin of the $X, Y$ coordinate system at the arbitrarily chosen initial instant $\xi=0$, maintaining the constant axial velocity $U_{0}$ and simultaneously translating vertically, with vertical velocity at the center of gravity $v_{c}(\xi)$ being an arbitrary function of time $\xi$. The angle of attack $\alpha$ is defined to be the angle between the resultant velocity vector and the aircraft's longitudinal axis, so that $\alpha=\tan ^{-1}\left[v_{c}(\xi) / U_{0}\right]$. We show first how a form for the response in $C_{L}$ to the arbitrary variation in a can be constructed from a suitable summation of lift responses to pulses in $a$. 
II.1. Nonlinear algebraic functional expansion.

Let the angle of attack a be zero for all time $\xi$ except at $\xi=\xi_{1}$, where a pulse occurs of amplitude $a\left(\xi_{q}\right)$ and of infinitesimal duration $\Delta \xi_{1}$. Consider the response to the pulse at $\xi_{1}$ of the lift coefficient $C_{L}$ at a measuring time $t$ subsequent to $\xi_{1}$. The lift response at $t$ will be a function of the elapsed time $t-\xi_{1}$, and the amplitude of the pulse $\alpha\left(\xi_{1}\right)$. If we assume that there will be a range of $\alpha$ around $\alpha=0$ in which the dependence of $C_{L}$ on $\alpha\left(\xi_{1}\right)$ is analytic, then we can write the response in $C_{L}$ as a Taylor-series expansion in $\alpha\left(\xi_{q}\right)$ of the form

$$
\Delta C_{L}(t)=\Delta C_{L}\left(t-\xi_{1}, \alpha\left(\xi_{1}\right)\right)_{d i r}=\sum_{n} a_{n}\left(t-\xi_{1}\right)\left[a\left(\xi_{1}\right)\right]^{n} \Delta \xi_{1}
$$

The first of the forms in (1) will be used to distinguish between direct (subscript dir) and interference (subscript int) effects.

Now let us consider the response $C_{L}$ at the measuring time $t$ to a pair of pulses located at $\xi_{1}$ and $\xi_{2}$ with $\xi_{1}, \xi_{2}<t$. In addition to the direct influence of each of the pulses acting as if in isolation, the interference between the pulses also will influence the lift. The interference effect can be written in a form resembling a product of responses to single pulses

$$
\Delta^{2} C_{L_{i n t, 2}}=\sum_{m, n} b_{m n}\left(t-\xi_{2}, \xi_{2}-\xi_{1}\right)\left[\alpha\left(\xi_{1}\right)\right]^{n}\left[\alpha\left(\xi_{2}\right)\right]^{m} \Delta \xi_{1} \Delta \xi_{2}
$$

where the subscript (int,2) means "interference between a pair of pulses." The form vanishes properly with the vanishing of either of the pulses and retains the analytic dependence on angle of attack. With the addition of the 
direct influence of the two pulses, the lift coefficient at time $t$ takes the form

$$
\Delta C_{L}(t)=\Delta C_{L}\left(t-\xi_{1}, a\left(\xi_{1}\right)\right)_{d i r}+\Delta C_{L}\left(t-\xi_{2}, \alpha\left(\xi_{2}\right)\right)_{d i r}+\Delta C_{L_{\text {int }, 2}}
$$

The process of adding pulses can be continued indefinitely in the same way. At the next stage, the interference between triplets of pulses must be considered as well as that between pairs. In the limit of a continuous distribution of pulses starting in the distant past, a summation of multiple integrals is obtained having the form*

$$
C_{L}(t)=C_{L_{\text {dir }}}+C_{L_{\text {int }, 2}}+C_{L_{\text {int }, 3}}+\cdots
$$

with

$$
\begin{gathered}
C_{L_{d i r}}=\sum_{n} \int_{-\infty}^{t} a_{n}\left(t-\xi_{1}\right)\left[\alpha\left(\xi_{1}\right)\right]^{n} d \xi_{1} \\
C_{L_{i n t, 2}}=\sum_{m, n} \int_{-\infty}^{t}\left[\alpha\left(\xi_{2}\right)\right]^{m} d \xi_{2} \int_{-\infty}^{\xi_{2}} b_{m n}\left(t-\xi_{2}, \xi_{2}-\xi_{1}\right)\left[\alpha\left(\xi_{1}\right)\right]^{n} d \xi_{1}
\end{gathered}
$$

*In [11] we verified that the form is consistent with a solution of the Navier-Stokes equations. 


$$
\begin{aligned}
c_{L_{\text {int }, 3}=} & \sum_{m, n, p} \int_{-\infty}^{t}\left[\alpha\left(\xi_{3}\right)\right]^{m} d \xi_{3} \int_{-\infty}^{\xi_{1}}\left[\alpha\left(\xi_{2}\right)\right]^{n} d \xi_{2} \\
& \times \int_{-\infty}^{\xi_{2}} c_{m n p}\left(t-\xi_{3}, \xi_{3}-\xi_{2}, \xi_{2}-\xi_{1}\right)\left[\alpha\left(\xi_{1}\right)\right]^{p} d \xi_{1}
\end{aligned}
$$

Equation (4) represents the lift coefficient at time $t$ in response to an arbitrary variation of $a$ over the time interval $-\infty<\xi<t$. It has essentially the same form as the nonlinear algebraic functional expansion advocated in [9] except that it can accommodate a slightly more general initial state. The form confirms an important point made in [9]. A partial summation consisting of the leading term from each of $(5),(6), \ldots$ will be seen to form a Volterra series [12]. The existence of additional terms is a confirmation that the a priori adoption of a Volterra series to represent the lift coefficient would not have bee: sufficiently general to accommodate the Taylor series form of the dependence on angle of attack.

II.2. An alternative functional expansion.

The following alternative form of functional expansion has proven particularly useful in application to problems in flight dynamics. There, it is easy to justify a simplifying approximation that is valid on the assumption of a slowly varying motion.

In general, the lift response $C_{L}$ at time $t$ is a functional [12] of the motion history $a(\xi)$ over the full time-interval $-\infty<\xi<t$ : 


$$
C_{L}(t)=C_{L}[a(\xi)]
$$

If $\alpha$ can be considered an analytic function of $\xi$, its history can be reconstructed, in principle, from a knowledge of all of the coefficients of its Taylor-series expansion about $\xi=t$. Since $\alpha(\xi)$ is equally represented by the coefficients of its expansion, it follows that the functional, with its dependence on $\alpha(\xi)$, can be replaced without approximation by a function which depends on all of the coefficients of the expansion of $\alpha(\xi)$ at $\xi=t$ :

$$
C_{L}[\alpha(\xi)]=C_{L}(\alpha(t), \alpha(t), \ddot{\alpha}(t), \ldots)
$$

Assuming now that $\alpha(t)$ is potentially large but that the rates $\dot{\alpha}(t), \ddot{\alpha}(t), \ldots$ are all always small, we are permitted to expand $(9)$ around the zero values of the rates, so that.

$$
\begin{aligned}
C_{L}(t)= & C_{L}[\alpha(\xi)]=C_{L}(\alpha(t), 0,0, \ldots)+\dot{\alpha}(t) C_{L_{\dot{\alpha}}}(\alpha(t), 0,0, \ldots) \\
& +\frac{\dot{\alpha}^{2}(t)}{2} C_{L_{\ddot{\alpha} \dot{\alpha}}(\alpha(t), 0,0, \ldots)+\ldots} \\
& +\ddot{\alpha}(t) C_{L_{\alpha} \cdot(\alpha(t), 0,0, \ldots)+\ldots}(x \\
& +\ldots
\end{aligned}
$$

This is an alternative form of functional expansion which is compatible with (4). Compatibility can be verified if each of the terms involving $\left[\alpha\left(\xi_{i}\right)\right]^{q}$ in the integral terms in (4) is expanded about the value of $\xi_{i}$ at its upper limit. Then the integrations can be carried out yielding the form 
(11) $C_{L}(t)=\sum_{n} A_{n}[\alpha(t)]^{n}+\dot{\alpha}(t) \sum_{n} B_{n}[\alpha(t)]^{n}+\dot{\alpha}^{2}(t) \sum_{n} C_{n}[\alpha(t)]^{n}+\cdots$

which can be identified term by term with (10).

The usefulness of the form of $(10)$ becomes apparent when we invoke the idea of a fading memory, which justifies discarding rate terms beyond the first-order term involving $\dot{a}(t)$. The basis of the idea is physical. We argue that the lift response $C_{L}$ at time $t$ should have "forgotten" longpast events, and so should depend mainly on events in the most recent past. On this assumption, so far as the lift response is concerned, the form of the past motion just prior to $\xi=t$ might just as well have existed for all earlier times. Hence at most, only the first few terms of the expansion of $\alpha(\xi)$ need be retained to characterize correctly the most recent past, which, on the assumption, is all that the lift response at $t$ remembers. Retaining the first two coefficients of $\alpha(\xi)$, for example, implies matching the true past history of $a$ in magnitude and slope at $\xi=t$, thereby approximating $\alpha(\xi)$ by a linear functior of time $\alpha(\xi) \approx \alpha(t)+(t-\xi) \dot{\alpha}(t)$. The idea has been given a firm mathematical basis in an important paper by Coleman and Noll [13]. Invoking $1 t$, we have as a representation of $C_{L}(t)$ for slowly varying motions

$$
C_{L}(t)=C_{L}(\alpha(t), 0,0, \ldots)+\dot{\alpha}(t) C_{L_{\dot{\alpha}}}(\alpha(t), 0,0, \ldots)
$$

The representation has played an important role in flight dynamics studies because it allows decoupling of the mathematical modeling problem for the aerodynamic contribution from the aircraft's equations of motion. On the basis of (12), the aerodynamic coefficients can be evaluated once and for all and be available without change for a range of possible motions. The notation 
itself in (12) suggests the types of experiments that could be performed to evaluate the coefficients. The first term $C_{L}(a(t), 0,0, \ldots)$ is the lift coefficient that would be measured at time $t$ for a steady motion in which the angle of attack had remained constant at the instantaneous value $\alpha(t)$ for all time prior to $t$. One should note, however, that nothing requires the coefficient itself to remain constant. In fact, in a later section we shall see that vortex-shedding can cause the coefficient to be a periodic function of time. The second term $C_{L_{\dot{\alpha}}}(\alpha(t), 0,0, \ldots)$ is the rate of change with $\dot{a}$, evaluated at $\dot{a}=0$, of the lift coefficient that would be measured for $a$ motion in which the angle of attack had varied linearly with time up to the present instant at which its value is $a(t)$. However, again within the assumption that motion in the distant past has been forgotten, any of a class of sufficiently slowly varying motions which arrive at the same value of $\alpha$ at time $t$ will yield the same value of $C_{L_{\dot{\alpha}}}$.

II.3. Indicial response and generalized superposition integral.

Finally, we shall show that our use of indicial responses to form a general integral representation of the aerodynamic response is compatible with the representation of the aerodynamic response based on nonlinear algebraic functional expansions given by (4). Let us begin by using (4) to form the indicial response according to our definition of it. To indicate the form of the result, it will suffice to consider terms in (4) only through the series representing $\mathrm{C}_{\mathrm{L}_{\text {int, }}}$. Two motions need to be considered ( $\mathrm{cf}$. Fig. 2). In the first, the aircraft undergoes the motion under study $a(\xi)$ from time $\xi \rightarrow-\infty$ up to a time $\xi=\tau$, where $\tau<t$. Subsequent to $\tau$, $\alpha$ is held constant at $a(\tau)$. Thus, in (4), (5), and (6), 


$$
\begin{array}{rlrl}
\alpha_{1}(\xi) & =\alpha(\xi) ; & & -\infty<\xi<\tau \\
& =\alpha(\tau) ; & \xi \geq \tau
\end{array}
$$

The direct and interference contributions to $C_{L}(t)$ take the form

$$
(14)\left\{\begin{aligned}
C_{L_{d i r}}= & \sum_{n} \int_{-\infty}^{\tau} a_{n}\left(t-\xi_{1}\right)\left[\alpha\left(\xi_{1}\right)\right]^{n} d \xi_{1}+\sum_{n}[\alpha(\tau)]^{n} \int_{\tau}^{t} a_{n}\left(t-\xi_{1}\right) d \xi_{1} \\
C_{L_{i n t, 2}} & \sum_{m, n} \int_{-\infty}^{\tau}\left[\alpha\left(\xi_{2}\right)\right]^{m} d \xi_{2} \int_{-\infty}^{\xi_{2}} b_{m n}\left(t-\xi_{2}, \xi_{2}-\xi_{q}\right)\left[\alpha\left(\xi_{1}\right)\right]^{n} d \xi_{1} \\
& +\sum_{m, n}[\alpha(\tau)]^{m} \int_{\tau}^{t} d \xi_{2} \int_{-\infty}^{\tau} b_{m n}\left(t-\xi_{2}, \xi_{2}-\xi_{1}\right)\left[\alpha\left(\xi_{1}\right)\right]^{n} d \xi_{1} \\
& +\sum_{m, n}[\alpha(\tau)]^{m+n} \int_{\tau}^{t} d \xi_{2} \int_{\tau}^{\xi_{2}} b_{m n}\left(t-\xi_{2}, \xi_{2}-\xi_{1}\right) d \xi_{1}
\end{aligned}\right.
$$

In functional notation [12], the lift response to the same motion is

$$
C_{L}(t)=C_{L}\left[a_{1}(\xi)\right]=C_{L}[a(\xi) ; t, \tau]
$$

where the notation in the first form should be understood to mean that $C_{L}(t)$ is a functional of the variation $\alpha_{1}(\xi)$ over the interval $-\infty<\xi<t$. The second form is intended to make more explicit that $\alpha_{1}(\xi)$ represents the motion under study $a(\xi)$ over the interval $-\infty<\xi<\tau$, but is constrained to remain constant at $\alpha(\tau)$ for $\tau \leq \xi \leq t$. Thus $C_{L}(t)$ is both a functional of $a(\xi)$ and a function of $t$ and $\tau$.

In the second motion, the aircraft undergoes the same angle-of-attack history $\alpha(\xi)$ up to time $\tau$. Subsequent to $\tau$, the angle of attack is again held constant, but is given an incremental step change $\Delta a$ over its previous value of $\alpha(\tau)$. Thus, in the second motion, 


$$
\begin{array}{rlrl}
\alpha_{2}(\xi) & =\alpha(\xi) ; & & -\infty<\xi<\tau \\
& =\alpha(\tau)+\Delta \alpha ; \quad \xi \geq \tau
\end{array}
$$

The direct and interference contributions to $c_{L}(t)$ become

$$
\left\{\begin{aligned}
c_{L_{d i r}}= & \sum_{n} \int_{-\infty}^{\tau} a_{n}\left(t-\xi_{1}\right)\left[\alpha\left(\xi_{1}\right)\right]^{n} d \xi_{1}+\sum_{n}[\alpha(\tau)+\Delta \alpha]^{n} \int_{\tau}^{t} a_{n}\left(t-\xi_{1}\right) d \xi_{1} \\
c_{L_{i n t, 2}}= & \sum_{m, n} \int_{-\infty}^{\tau}\left[a\left(\xi_{2}\right)\right]^{m} d \xi_{2} \int_{-\infty}^{\xi_{2}} b_{m n}\left(t-\xi_{2}, \xi_{2}-\xi_{1}\right)\left[\alpha\left(\xi_{1}\right)\right]^{n} d \xi_{1} \\
& +\sum_{m, n}[a(\tau)+\Delta \alpha]^{m} \int_{\tau}^{t} d \xi_{2} \int_{-\infty}^{\tau} b_{m n}\left(t-\xi_{2}, \xi_{2}-\xi_{1}\right)\left[\alpha\left(\xi_{1}\right)\right]^{n} d \xi_{1} \\
& +\sum_{m, n}[a(\tau)+\Delta \alpha]^{m+n} \int_{\tau}^{t} d \xi_{2} \int_{\tau}^{\xi_{2}} b_{m n}\left(t-\xi_{2}, \xi_{2}-\xi_{1}\right) d \xi_{1}
\end{aligned}\right.
$$

Again, written as a functional, the lift response to the motion $\alpha_{2}(\xi)$ is

$$
C_{L}(t)=C_{L}\left[\alpha_{2}(\xi)\right]
$$

The indicial lift response is formed by taking the difference between (14) and (17) (or between (15) and (18)), dividing by the incremental step $\Delta \alpha$, and going to the limit as $\Delta \alpha+0$. Existence of the limit is ensured by the analyticity of the functional dependence on $\alpha(\xi)$. The result is 
(19)

$$
\begin{aligned}
\lim _{\Delta \alpha \rightarrow 0} \frac{\Delta C_{L}(t)}{\Delta \alpha}= & \lim _{\Delta \alpha \rightarrow 0}\left(\frac{C_{L}\left[\alpha_{2}(\xi)\right]-C_{L}\left[\alpha_{1}(\xi)\right]}{\Delta a}\right) \\
= & \sum_{n} n[\alpha(\tau)]^{n-1} \int_{\tau}^{t} a_{n}\left(t-\xi_{1}\right) d \xi_{1} \\
& +\sum_{m, n} m[\alpha(\tau)]^{m-1} \int_{\tau}^{t} d \xi_{2} \int_{-\infty}^{\tau} b_{m n}\left(t-\xi_{2}, \xi_{2}-\xi_{1}\right)\left[\alpha\left(\xi_{1}\right)\right]^{n} d \xi_{1} \\
& +\sum_{m, n}(m+n)[a(\tau)]^{m+n-1} \int_{\tau}^{t} d \xi_{2} \int_{\tau}^{\xi_{2}} b_{m n}\left(t-\xi_{2}, \xi_{2}-\xi_{1}\right) d \xi_{1}
\end{aligned}
$$

Equation (19) reveals the form of the indicial lift response to a step change in angle of attack in terms of functional expansions. The first and third terms on the right side of (19) do not depend on the past motion, but only on the level of the angle of attack at which the step was made. The second term depends on the past motion since $\alpha\left(\xi_{\uparrow}\right)$ with $-\infty<\xi_{1}<\tau$ appears within the integral. The leading term of this past dependence has the form

$$
\int_{\tau}^{t} d \xi_{2} \int_{-\infty}^{\tau} b_{11}\left(t-\xi_{2}, \xi_{2}-\xi_{1}\right) \alpha\left(\xi_{1}\right) d \xi_{1}
$$

Dependence on the past thus arises from the interference effects between pulses prior to $\tau$, the origin of the step, and perturbation pulses of $O(\Delta a)$ originating subsequent to $\tau$. In the general case, then, the indicial response is itself a functional.

In fact, in formal terms (see [14] for a useful account of the essentials) the operations involved in forming the indicial response amount to taking a Fréchet derivative of the functional $C_{L}\left[\alpha\left(\xi_{1}\right)\right]$. To see this, we adopt the notation of [14], letting 
where we set

$$
\left\{\begin{array}{rlrl}
\varepsilon & =\Delta \alpha \\
\eta & =0 ; & -\infty<\xi<\tau \\
& =1 ; \quad \xi \geq \tau
\end{array}\right.
$$

so that

$$
\alpha_{2}(\xi)=\alpha_{1}(\xi)+\varepsilon \pi
$$

Then, following [14], we have

$$
\begin{aligned}
\lim _{\Delta \alpha \rightarrow 0} \frac{\Delta C_{L}(t)}{\Delta \alpha} & =\lim _{\varepsilon \rightarrow 0}\left(\frac{C_{L}\left[\alpha_{1}(\xi)+\varepsilon n\right]-C_{L}\left[\alpha_{1}(\xi)\right]}{\varepsilon}\right) \\
& =\left.\frac{d}{d \varepsilon} C_{L}\left[\alpha_{1}(\xi)+\varepsilon \eta\right]\right|_{\varepsilon=0} \\
& =C_{L}^{\prime}\left[a_{1}(\xi)\right] n
\end{aligned}
$$

The equivalence of the operations yielding (19) and (23) should be evident. Finally, to conform with the second form in (15), we set

$$
\lim _{\Delta a \rightarrow 0} \frac{\Delta C_{L}(t)}{\Delta \alpha}=C_{L}^{\prime}\left[\alpha_{1}(\xi)\right] n \equiv C_{L_{\alpha}}[\alpha(\xi) ; t, \tau]
$$

where the indication of a separate dependence on $t$ and $\tau$ rather than on elapsed time $t-\tau$ alone should be noted. The first and third terms in 
(19), which depend only on $\alpha(\tau)$, indicate a dependence on $t-\tau$ alone as can be easily verified; however, as a consequence of its dependence on the past motion, the second term cannot be cast as a function of $t-\tau$ alone. Equation (24) can now be used to form a generalized superposition integral for the response in $C_{L}$ to an arbitrary angle-of-attack variation. The result is

$$
C_{L}(t)=\int_{-\infty}^{t} C_{L_{\alpha}}[\alpha(\xi) ; t, \tau] \frac{d \alpha}{d \tau} d \tau
$$

Equivalently, to make explicit the dependence on an initial state, (25) may be rewritten as

$$
C_{L}(t)=C_{L}[\alpha(\xi) ; t, 0]+\int_{0}^{t} C_{L_{\alpha}}[\alpha(\xi) ; t, \tau] \frac{d \alpha}{d \tau} d \tau
$$

Note that the first term in $(26)$ is the lift at time $t$ resulting from an angle-of-attack variation $\alpha(\xi)$ which is equal to the motion history prior to $\xi=0$, and which is constrained at $\xi=\tau=0$ to remain constant at $\alpha(0)$ for all $\xi \geq \tau=0$.

By substituting (19) for $\mathrm{C}_{\mathrm{L}_{\alpha}}[\alpha(\xi) ; t, \tau]$ in (25) and carrying out the integration, one will verify that the form of (4) is restored through terms of the series representing $C_{L_{\text {int,2 }}}$ (i.e., through (5) and (6)). We conclude first that an approach to modeling based on nonlinear indicial responses and generalized superposition integrals is compatible with one based on nonlinear algebraic functional expansions. Second, to the extent that the three representations discussed in this section are compatible, their validity rests on the Fréchet differentiability of the lift response $C_{L}[a(\xi) ; t, t]$ over the 
interval $-\infty<\xi<t$. In a later section, we shall take up the means by which Eréchet differentiability of the 1 ift response may be invalidated.

III. QUALIFICATIONS

Before proceeding with the study of Fréchet differentiability, we must Introduce some important qualifications. Our current understanding of the fluid-dynamic aspects of the problem stems almost entirely from results of studies of laminar flows governed by the incompressible Navier-Stokes equations. Within these confines, studies have been further limited to flows in bounded domains. Boundary conditions have been simple enough to permit reduction of the linear stability problem (the necessary first step in the study of bifurcations) to the form of ordinary differential equations (e.g., the OrrSommerfeld equations). The principal findings on which our current understanding is based are summarized in [15-18]. Qualifications are necessary when we attempt to extrapolate this body of knowledge to the study of fluiddynamic aspects under the conditions of full-scale flight. These involve turbulent compressible flows, which, if averaging and modeling are introduced, will be governed not by the compressible Navier-Stokes equations but by evolution equations of unknown forms. Further, the domains of the flows are typically unbounded, rather than bounded. Finally, the boundary conditions, reflecting the geometries typical of modern aircraft, ordinarily will not allow reduction of the linear stability problem to the level of ordinary differential equations.

The situation requires us to stipulate a list of presuppositions which will be the basis of the extrapolations we make. The list is inspired by one that Lanford compiled to justify the study of turbulence within a framework 
based on the incompressible Navier-Stokes equations [19]. Our 1ist will merely supplement Lanford's, taking additional account of turbulence modeling, compressibility, and the necessity to consider flows in unbounded domains. The issue of boundary conditions is not addressed on the assumption that their increased complexity does no more than enlarge the role of numerical computation.

III. 1. Turbulence modeling.

Let us consider the flow field to which a typical lift response $C_{L}[\alpha(\xi) ; t, \tau]$ corresponds. We assume that the flow field at time $\xi=\tau$ is given (to within a certain accuracy) and we must determine the subsequent flow at time $t$. Inasmuch as $\alpha(\xi)$ remains constant at $\alpha(\tau)$ for all $\xi=t>\tau$, we have fixed boundary conditions. This form of the fluid-dynamics problem is known as an initial boundary value problem (IBVP; cf. [16]). We assume that any given realization of the IBVP is governed with sufficient accuracy by the compressible Navier-Stokes equations. Paralleling [19], in the absence of proof we simply postulate that a solution of the IBVP would exist for all $\xi=t \geq \tau$ and would be unique. However, the Reynolds number is sufficiently high that a solution would exhibit chaotic behavior, Implying a sensitive dependence on the initial conditions. Thus, small perturbations of the initial conditions would cause repetitions of the given motion to result in a set of solutions having stochastic properties.

The situation calls for the application of some form of averaging of the compressible Navier-Stokes equations to suppress the appearance of the smallscale chaotic structures. After averaging, the repertory of solutions of the resulting equations must remain faithful to that of the unaveraged equations, 
neither allowing uncharacteristic solution forms nor losing essential ones. The necessity of closing the averaged equations in conformity with this requirement introduces the turbulence modeling problem. We cannot go further here in specifying modeling requirements except to postulate the one feature that must be retained in common with the original Navier-Stokes equations: Whatever the form of modeling adopted, it must remain possible to cast the modeled equations in the form of autonomous evolution equations, namely,

$$
\vec{u}_{t}=H(\vec{u}, \lambda)
$$

where $\overrightarrow{\mathrm{u}}$ is the velocity vector and $\lambda$ is a parameter (e.g., angle of attack, Reynolds number, Mach number). We believe that the premise is a reasonable one, involving no more in principle than the use of orthogonal projections $[20,21]$ to eliminate gradient terms (e.g., the pressure term) from the modeled equations.

\section{III.2. Compressibility .}

We shall assume that modeled equations of turbulent flow resulting from averaging the compressible Navier-Stokes equations remain at least as wellbehaved as the original equations and their incompressible counterparts. Of course, the new parameters introduced by compressibility (e.g., Mach number) will themselves have critical values corresponding to additional mechanisms by which Fréchet differentiability may be invalidated. 
III.3. Unbounded domains.

Let us return to the IBVP represented by $C_{L}[\alpha(\xi) ; t, \tau]$ and assume that the premises of the previous sections are now in force. Henceforth, the equations governing the flow field are assumed to be modeled equations of turbulent flow, of known form, and that satisfy the form of (27) by suitable projections.

The issue concerning the absence of bounds on the flow domain arises in evaluating the stability of the flow field in the equilibrium state. We say that the equilibrium state is reached in the limit as $t-\tau \rightarrow \infty$, so that, presumably, transient effects associated with the initial conditions have vanished. Bifurcation of the equilibrium state is one means by which Fréchet differentiability of the lift response may be invalidated. To investigate the possibility, a study of the stability of the equilibrium state must be undertaken first, since bifurcation of the equilibrium state will not occur unless a critical value of the parameter in question (here, a) exists at which the flow field in the equilibrium state becomes unstable. Stability is evaluated by determining whether an infinitesimal perturbation of the equilibrium flow decays or grows with time. The perturbation's fate may be determined from a linear spectral problem; the governing equations are obtained from a linearized perturbation of the equations governing the equilibrium flow. Typically, with a bounded domain, it can be shown that solution of the spectral problem yields a countably infinite set of isolated eigenvalues in a complex plane. Stability is indicated if all of the eigenvalues lie in the left half of the plane. On the other hand, little is known about the spectrum of eigenvalues for unbounded domains. In the case of flows in unbounded domains governed by the incompressible Navier-Stokes equations, it is believed $[22,23]$ that an 
incomplete set of discrete eigenvalues exists which again controls stability, and that there is additionally a continuous spectrum having negative real part (i.e., lying in the left half of the complex plane). We postulate that what is believed to be the case for flows in unbounded domains governed by the incompressible Navier-Stokes equations is in fact the case for our problem.

\section{III.4. Bifurcation theory.}

Accepting the last premise (which implies accepting all of the previous ones) leads to several conclusions concerning the role of bifurcation theory in the modeling of aerodynamic responses. First, since we have postulated that stability of the equilibrium state continues to be controlled by the discrete part of the eigenvalue spectrum alone, entailing no change from the rules governing bounded domains, we conclude that the role of bifurcation theory regarding the equilibrium state should carry over intact to flows in unbounded domains. That is, all of the instability mechanisms that have been uncovered by studies of flows in bounded domains governed by the incompressible Navier-Stokes equations should have counterparts in flows in unbounded domains governed by modeled turbulent-flow equations. With the onset of instability of the equilibrium state, bifurcation theory again should be capable of classifying and characterizing the properties of the new stable equilibrium states that can arise to replace the unstable one.

On the other hand, since bifurcation theory is concerned only with the equilibrium state, it does not suffice to completely resolve the IBVP from which the indicial response is derived. We note that when the eigenvalue spectrum lies in the left half of the plane, indicating stability of the equilibrium state, the IBVP for an infinitesimal step perturbation of the 
boundary condition $a(\xi)=a(\tau) ; \xi \geq \tau$ is a linear problem. In fact, the transient flow field from which the indicial response $C_{L_{\alpha}}[\alpha(\xi) ; t, \tau]$ is derived can be obtained from a suitable superposition of the eigensolutions (including both the discrete and continuous parts) of the linear spectral problem. The superposition is made determinate by the necessity of matching a prescribed flow field at the initial instant $\xi=\tau$. This is how the dependence on the past motion is expressed, which makes $C_{L_{\alpha}}[\alpha(\xi) ; t, \tau]$ a nonlinear functional even though it is derivable from linearized perturbation equations. Thus, recalling the derivation by means of functional expansions, we affirm that so long as stability of the equilibrium state is ensured, and so long as there are no other means available of invalidating Fréchet differentiability of the lift response $C_{L}[\alpha(\xi) ; t, \tau]$, then the differential $\Delta \alpha C_{L_{\alpha}}[\alpha(\xi) ; t, \tau]$ can be determined from a linear problem. With the onset of instability, however, the perturbation flow no longer can be obtained from the solution of the linear spectral problem, since at least one of the discrete eigensolutions would grow without bounds. Then, the incremental change in lift coefficient must be determined from the fully nonlinear perturbation flow equations.

IV. FRÉCHET DIFEERENTIABILITY

Referring to Figure 3, let us assume that the angle-of-attack variation under study $\alpha(\tau)$ passes through a critical value $a_{c}$ at a value of $\tau=\tau_{c}$ within the interval of interest, $0<\tau<t$. We say that $\alpha_{c}$ is a critical value in this sense: If the angle-of-attack variation $\alpha(\tau)$ were constrained to remain constant at $a_{c}$ for all $\tau>\tau_{c}$, the corresponding lift response $C_{L}\left[\alpha(\xi) ; t, \tau_{c}\right]$ would begin to depart from an initially infinitesimally close 
neighboring response, such that $C_{L}\left[a(\xi) ; t, \tau_{c}\right]$ would not be Fréchet differentiable. Thus, we cannot allow the integration in (26) to proceed beyond ${ }^{c} c$ without acknowledging the loss of Fréchet differentiability. Let us assume that for all other angles of attack within the range $\alpha(0)<\alpha<\alpha(t)$, the lift responses permit Fréchet differentiation. Then we can isolate the critical state by stopping the integration in (26) just short of $\tau_{c}$ and starting again fust beyond $\tau_{c}$. Within the isolated interval $\tau_{c}-\varepsilon<\tau<\tau_{c}+\varepsilon$, we acknowledge the loss of Fréchet differentiability by allowing the solution to change discretely to a new state. Thus,

$$
\begin{aligned}
C_{L}(t)= & C_{L}[\alpha(\xi) ; t, 0]+\int_{0}^{\tau} c^{-\varepsilon} C_{L_{\alpha}}[\alpha(\xi) ; t, \tau] \frac{d \alpha}{d \tau} d \tau \\
& +\int_{\tau_{c}+\varepsilon}^{t} C_{L_{\alpha}}[\alpha(\xi) ; t, \tau] \frac{d \alpha}{d \tau} d \tau+\Delta C_{L}\left(t ; \alpha_{c}\right)
\end{aligned}
$$

where

$$
\Delta C_{L}\left(t ; \alpha_{C}\right)=C_{L}\left[\alpha(\xi) ; t, \tau_{C}+\varepsilon\right]-C_{L}\left[\alpha(\xi) ; t, \tau_{C}-\varepsilon\right]
$$

As we have just noted for the case of bifurcation, the incremental change in the lift coefficient represented by (29) must be determined from the fully nonlinear perturbation flow equations.

We have argued [10] that a theory for enumerating the means of invalidating Fréchet differentiability of the aerodynamic response may be a more inclusive way than bifurcation theory of classifying steady and unsteady aerodynamic phenomena that are important in flight-dynamics applications. At present we have identified three distinct means. 
IV.1. Aerodynamic bifurcation.

We define aerodynamic bifurcation as the replacement of an unstable equilibrium flow by a new stable equilibrium flow at a critical value of a parameter. The onset of instability of the equilibrium state is a linear problem that is capturable, at least in principle, through analysis of a linearized form of the time-dependent modeled equations of turbulent flow.

An example that illustrates aerodynamic bifurcation in a context relevant to current interests is the slender body of revolution at a high angle of attack. We shall describe this problem heuristically by adapting the impulsive flow analogy, both to demonstrate the continued usefulness of the analogy and to make a point that will be important later. The analogy has been used extensively to help explain the presence of the steady symmetric vortices that are found on the leeward of slender wings and bodies at high angles of attack. To review briefly, we refer to Figure 4 where now the moving axis system $(x, r, \theta)$ is fixed to the body nose and the body is sinking with uniform velocity $v_{c}$. On a vertical plane fixed in space, e.g., the plane $x=0$, if the growth of the body is ignored, one observes the flow about a cylinder that appears to have started from rest and is sinking at the uniform rate $v_{c}$. On the assumption that the flow in the vertical plane is essentially twodimensional, one is able to relate the steady growth of the vortices in the body-axis system with distance $x$ along the body to their time-dependent growth behind the impulsively started cylinder, as viewed in the $X=0$ plane. The latter problem has been studied extensively both experimentally [24] and via numerical computation [25]. Thorough documentation exists for the first appearance of separation on the leeward ray, followed by the growth of the primary vortices, then the secondary vortices, and so forth. Also, it 
is known that beyond a critical Reynolds number ( $R e \approx 50$ based on $v_{c}$ and on body diameter), the equilibrium flow becomes time-dependent, leading to the periodic shedding of vortices in the wake. This phenomenon also has been studied extensively. See especially the work of Nishioka and Sato [26], which demonstrates clearly that the onset of periodicity occurs at a critical Reynolds number, an event which is, in effect, a Hopf bifurcation. We expect, then, that at a critical Reynolds number, a perturbation velocity within a fixed plane will have behavior that can be expressed initially as

$$
v=g(r, \theta) e^{i \omega t}
$$

where if $v$ is radial velocity, then $g(r, \theta)$ is antisymmetric with respect to $\theta=0$. Now, we have assumed that the axial flow induced by the cylinder as it moves through the plane is more or less independent of $X$. A nominally two-dimensional flow will permit a wave-form perturbation solution in the direction of the third coordinate. A more general form of the perturbation velocity $v$ is then

$$
v=g(r, \theta) e^{i \omega t} e^{i \lambda X}
$$

Transforming this solution to the body-axis system with $X=x-U_{0} t$, we obtain

$$
v=g(r, \theta) e^{i \lambda\left[x-U_{0} t+(\omega / \lambda) t\right]}
$$

and we see that a stationary perturbation solution is possible in the bodyaxis system when the wave speed $\omega / \lambda$ equals the axial speed $U_{0}$. 
Experimental results indicate that a stationary asymetric flow is the preferred solution over a considerable range of angle of attack. We conjecture that it is easy for an equality between $U_{0}$ and the wave speed $\omega / \lambda$ to be achieved for the following reason. Owing to the presence of the vortices, the axial velocity profile has a considerable overshoot beyond the freestream value $U_{0}$. The wave speed $\omega / \lambda$ must 1 ie between the extremes of the axial velocities available and hence can easily match $U_{O}$. On the other hand, as $\alpha+90^{\circ}, \lambda+0$, so a match becomes impossible, and the perturbation form reverts to (30) in either axis system.

The analysis leads to the following conclusions. 1) Having demonstrated its ability to fit observations, the impulsive flow analogy remains a useful conceptual device for understanding complex three-dimensional flows.

2) Accepting 1) encourages the belief that instabilities in complex threedimensional flows may be essentially local phenomena which will yield to localized analyses. 3) The form of bifurcation, and hence its classification, may depend on the coordinate system in which it is observed. Fréchet differentiability on the other hand, remains invariant across coordinate transformations.

IV.2. Loss of analytic dependence on a parameter.

The following refers principally to time-invariant equilibrium flows. The variation with a parameter (e.g., roll angle $\psi$ ) of the aerodynamic response may develop a fold at a critical value of the parameter $\psi_{c}$, so that the slope of the response becomes infinite there. A jump in the response necessarily ensues to another branch of the folded curve with an infinitesimal increase in $\psi$ beyond $\psi_{c}$, and hysteresis follows on the return route. Folds 
need not be the result of bifurcation; no new branches of equilibrium flow solutions need appear. On the other hand, folds may be the indirect result of bifurcation. An example based on the preceding study is the slender delta wing. Just as for the slender body of revolution, bifurcation to an asymmetric flow pattern occurs at a critical value of $a$, beyond which a finite value of the rolling-moment coefficient $C_{\ell}$ may exist at zero roll angle. As shown in Figure 5, both a positive and a negative value of $C_{\ell}$ are possible, depending on the sense of the asymmetry. The solution $c_{\ell}=0$, corresponding to the unstable symmetric flow, also exists at $\psi=0$, but is itself unstable. So folds must occur to accommodate the three solution points. We note that there is another important way in which time-invariant equilibrium flows may experience a loss of analytic dependence on a parameter: a change in flow topology. Changes in flow topology are defined unambiguously by changes in the number of singular points either in the pattern of surface skin-friction lines or in the external flow. An example is shown in Figure 6 taken from [27]. The line of primary flow separation on a body of revolution at a relatively low angle of attack (Fig. 6(a)) curves over smoothly from a position characteristic of a laminar boundary-layer flow to a position characteristic of a turbulent boundary-layer flow. At a higher angle of attack (Fig. 6(b)), the laminar line of separation terminates at a singular point (a focus) and the line begins again at the position characteristic of a turbulent boundary-layer flow. We anticipate that where such a change in flow topology first occurs (i.e., at a critical angle of attack), not only will the equilibrium lift response cease to be an analytic function of $\alpha$, but there will be a significant increase in the time required for the lift response to reach a new equilibrium state. If the (potentially discontinuous) increment in lift response yields an increment in pitching moment of the proper sign, 
this, coupled with the potentially large time lag, creates conditions for the sudden appearance of a potentially large destabilizing aerodynamic damping moment.

Heretofore, we have directed our extensive studies of flow topology [28-30] toward gaining an understanding of steady three-dimensional separated flows. We see here that the study of flow topology may have an important role to play in the context of flight dynamics studies as well.

\section{IV.3. Disconnected bifurcation.}

We define disconnected bifurcation as the existence of an isolated branch of equilibrium solutions that is not connected to other solution branches at bifurcation points. The sources of this form of invalidation are still obscure although evidence abounds of its existence in experimental studies. It is clear that the state of the flow at the origin of the indicial response $\xi=\tau$ must be involved. Disturbances there must be large enough and of an appropriate form so as to divert the response toward a new attractor, that is, toward an alternative branch of equilibrium solutions.

As we have already seen, a source to which nominally two-dimensional flows should be particularly susceptible is the presence of three-dimensional disturbances in cellular patterns in the direction of the third coordinate. As candidates for study of this situation, we cite two possibilities. The first one concerns flow at the stagnation line of a cylindrical body. The presence of a regular pattern of disturbances along, e.g., the leading edge of a straight wing has been known for a long time. Attempts to explain the pattern's presence on the basis of stability theory have been neither consistent nor convincing. In a comprehensive and useful review of the subject, 
Morkovin [31] concludes that there is still no viable explanation of the phenomenon. The second possibility could be axisymmetric vortex breakdown.* A solution here on the basis of a disconnected bifurcation would represent an extension to viscous flows of Brooke Benjamin's notion [32] of "conjugate flows" that he introduced as an aid to the explanation of inviscid axisymmetric vortex breakdown.

V. MEMORY EFFECTS

We have postulated six major subdivisions in the form of the aerodynamic response by means of a set of sketches [7], and these are reproduced in Figure 7. Of particular interest here are Figures $7(d)$ and $7(e)$, depicting time-dependent equilibrium states. Hopf bifurcation is indicated in Figure $7(d)$, wherein a formerly stable time-invariant equilibrium state is replaced by a time-varying periodic equilibrium state. The equilibrium state resulting from a Hopf bifurcation is often followed by bifurcation to a quasiperiodic equilibrium state with further increase of the relevant parameter (here, angle of attack). The quasi-periodic state may be succeeded by an aperiodic (chaotic) equilibrium state.

We indicated $(28,29)$ how these bifurcations could be accommodated within our mathematical model. However, in the implementation, an important additional issue first arises with a Hopf bifurcation when we recognize that it is necessary to specify phase as well as amplitude and frequency to completely determine the periodic equilibrium state. The general problem can be posed as follows: given that the past motion determines the initial condition for the

\footnotetext{
*Joint work with D. Weihs, Technion, Israel.
} 
specification of an indicial response, how much of the past motion must be acknowledged to ensure that the equilibrium state of the response is adequately specified? We see that there will be a definite sequence, in which at least one piece of additional information about the past motion must be supplied with each successive bifurcation. The sequence is illustrated in Figure 8. Let us note, incidentally, that with a linear system, no information about the past motion is required in the specification of an indicial response. This is, in effect, the most general definition of a linear system. For a nonlinear system in which the equilibrium state is time-invariant, the level of the past motion at the origin of the step is all that is required to specify the equilibrium state (Fig. 8(a)). For the time-periodic equilibrium state that replaces the time-invariant state with a Hopf bifurcation, the amplitude and frequency of the periodic state are again determined by the level of the past motion, but an additional piece of information, the rate of the past motion at the origin of the step must be supplied to fix the phase of the equilibrium state (Fig. $8(\mathrm{~b})$ ). If the next bifurcation results in a quasi-periodic equilibrium state, another derivative of the motion at the origin of the step will be required to specify the additional phase (Fig. 8(c)). It is clear that a chaotic equilibrium state will require information about the complete past motion to ensure a complete specification. This is how "sensitive dependence on the initial conditions," the signature of a chaotic state, will manifest itself in the modeling of the indicial response. It will be recalled that the equations governing the flow already have been postulated to be modeled equations of turbulent flow. The potential reemergence of chaotic behavior at a larger scale, even with modeled equations, suggests that re-modeled equations of turbulent flow may be required as well. 
We believe that our mathematical model, amended to accommodate the loss of Fréchet differentiability at isolated points $(28,29)$, should be capable of describing the aerodynamic characteristics in all six forms of aerodynamic force (or moment) responses illustrated in Figure 7 . The forms involving aerodynamic bifurcations that result in time-dependent equilibrium states (Figs. 7(d) and (e)) are of particular interest. Applied to problems involving dynamical maneuvers of aircraft, these forms will require extensive and novel experimentation. Problems in this category can be divided into two subclasses.

The first subclass of problems involves oscillatory motions about an equilibrium state at which a loss of Fréchet differentiability occurs. The case of dynamic stall of airfoils, which we studied at length in [10], typifies this subclass of problems. Similar cases need to be studied in which the oscillatory motions are free, rather than forced, to determine how vortexshedding frequencies may be modulated by the system's natural frequencies, causing, for example, "frequency lock-in." Another potentially important question is whether vortex-shedding (from, e.g., three-dimensional analogs of the airfoil's leading-edge separation bubble) may be a source of wing rock. There is considerable reason to belleve (cf., e.g., the experimental results reported in [33]) that such motions will have extensive regimes of chaotic behavior.

The second subclass of problems involves oscillatory motions that remain within a space free of critical values of parameters. Within such a space, in which the aerodynamic response can be assumed to remain Fréchet differentiable, simplifications in the modeling can be effected. In particular, the 
simple representation given by (12) can be applied with the additional assumption of a slowly varying motion. Dynamical systems within this subclass of problems involving vortex-shedding from obstacles have inspired a large collection of literature under the category of "vortex-induced oscillations." Excellent surveys of the field have been published by Sarpkaya [34] and more recently by Bearman [35]. The archetypal problem is the flexibly mounted cylinder immersed in a uniform oncoming stream. Our application of (12) to this problem yields a differential equation of the form*

$$
\ddot{h}+2 \mu \dot{h}+\kappa^{2} h=B(\dot{h}) \sin [\omega(\dot{h}) t]
$$

where the quantities $h, \mu, k$ characterizing the spring-mass system are illustrated in Figure $9(\mathrm{a})$. The forcing term, which expresses the contribution of the fluctuating lift caused by vortex-shedding, is novel in that the amplitude $B$ and frequency $\omega$ are coupled to the velocity $\dot{h}$ on the left side. Our modeling approach provides an explanation as follows: Amplitude $B$ is a function of instantaneous Reynolds number (Fig. $9(\mathrm{~b})$ ), which makes it a function of total velocity $\sqrt{u_{0}^{2}+\dot{h}^{2}}$. Strouhal number $k$, the dimensionless frequency of the fluctuating lift in the equilibrium state, is a constant, independent of Reynolds number in the range of velocities of interest (Fig. $9(b))$. This makes $w$, the actual frequency, a function of $\dot{h}$ via

$$
k=\frac{\omega d}{U_{\text {tot }}}=\frac{\omega d}{\sqrt{U_{0}^{2}+\dot{h}^{2}}}=\text { const }
$$

*The $\dot{a}$ term in (12) is discarded on the basis of its being proportional to vertical acceleration $\ddot{h}$, and hence normally negligible compared to the cylinder's inertial term. 
We have found that (33) has a rich repertory of solutions, and appears to be capable of capturing the distinctive features of the system's behavior (frequency lock-in, amplitude jumps, hysteresis) that careful experiments have revealed $[34,35]$. The rich repertory of $(33)$ is easier to understand if we transform it into a set of autonomous, first-order equations. There are three such equations, a number which suffices to permit solutions having chaotic behavior. Indeed, results of numerical computations over ranges of the parameters $B, k, \mu$, have revealed sequences of equilibrium states involving limit cycles (Lyapunov dimension $=1$; cf. [36] for a definition), 2-tori (Lyapunov dimension $=2$ ) and a strange attractor (Lyapunov dimension $=2.68$ ) .

VI. CONCLUDING REMARKS

This paper is a continuation of our effort to provide a consistent formulation and theoretical method for studying nonlinear problems in flight dynamics. Our study stressed a number of themes which led to the following conclusions:

1. Studies in nonlinear flight dynamics can be enhanced by linking them to the common features of parallel studies in various allied fields. The unifying medium is the rapidly growing body of theory underlying research in nonlinear dynamical systems. Exemplifying this, our approach to modeling, which involves nonlinear indicial responses and generalized superposition integrals, was made compatible with one originating in the field of electrical circuits and systems based on the use of nonlinear algebraic functional expansions. Operations involved in forming the indictal response were found to be equivalent to Fréchet differentiation. Invalidation of Fréchet differentiability proved to be the common element signaling the necessity of amending the 
mathematical model to accommodate potential discontinuous aerodynamic behavior.

2. Three means of invalidating Fréchet differentiability were identified: i) aerodynamic bifurcation, defined as the replacement of an unstable equilibrium flow by a new stable equilibrium flow at a critical value of a parameter; ii) loss of analytic dependence on a parameter; iii) disconnected bifurcation, defined as the existence of an isolated branch of equilibrium solutions not connected to other branches at bifurcation points. Acceptance of a list of presuppositions permitted us to extrapolate our understanding of these means from a framework involving bounded laminar flows governed by the incompressible Navier-Stokes equations to one involving unbounded turbulent flows governed by modeled equations based on the compressible Navier-Stokes equations. Amended to accomnodate the loss of Fréchet differentiability at isolated points, the mathematical model should be capable of describing the aerodynamic characteristics of all six major subdivisions that we have postulated for the aerodynamic response.

3. Accommodating bifurcation phenomena that involve time-dependent equilibrium states within the mathematical model was found to raise an issue of memory effects that becomes more important with each successive bifurcation. The implication is that with the emergence of a chaotic equilibrium state, information about the complete past motion may be required to ensure a complete specification of an indicial response.

4. Applied to the equation of motion governing the behavior of a flexibly mounted cylinder immersed in a uniform oncoming stream, our modeling approach yielded a novel form for the aerodynamic forcing term. Solutions of the equation captured distinctive features of the system's behavior (frequency lock-in, amplitude jumps, hysteresis). Results of computations over ranges of 
the parameters revealed sequences of equilibrium states involving limit cycles, 2-tori, and a strange attractor.

REFERENCES

[1] BRYAN (G. H.). Stability in aviation. MacMillan and Co., London (1911).

[2] JONES (B.). Dynamics of the aeroplane. Sec. $N$ of Aerodynamic Theory, 5, W. F. Durand, ed., Dover Publications, New York (1963).

[3] TOBAK (M.). On the use of the indicial function concept in the analysis of unsteady motions of wings and wing-tail combinations. NACA Rep. 1188 (1954).

[4] TOBAK (M.), PEARSON (W. E.). A study of nonlinear longitudinal dynamic stability. NASA TR R-209, USA (Sept. 1964).

[5] TOBAK (M.), SCHIFF (L. B.). On the formulation of the aerodynamic characteristics in aircraft dynamics. NASA TR R-456 (Jan. 1976).

[6] TOBAK (M.), SCHIFF (L. B.). Aerodynamic mathematical modeling--basic concepts. AGARD Lecture Series No, 114, Paper 1 (March 1981).

[7] CHAPMAN (G. T.), TOBAK (M.). Nonlinear problems in flight dynamics. Proc. Berkeley-Ames Conference on Nonlinear Problems in Control and Fluid Dynamics, Math Sci. Press (1985). Also NASA TM-85940 USA (May 1984).

[8] TOBAK (M.), CHAPMAN (G. T.), SCHIFF (L. B.). Mathematical modeling of the aerodynamic characteristics in flight dynamics. Proc. Berkeley-Ames Conference on Nonlinear Problems in Control and Fluid Dynamics, Math Sci. Press (1985). Also NASA TM-85880 USA (Jan. 1984).

[9] fLIESS (M.), LAMNAHBI (M.), LAMNAHBI $\angle$ LAGARRIGUE (F.). An algebraic approach to nonlinear functional expansions. IEEE Transactions on Circuits and Systems, 30, No. 8 (Aug. 1983), pp. 554-570. 
[10] TOBAK (M.), CHAPMAN (G. T.). Nonlinear problems in flight dynamics involving aerodynamic bifurcations. AGARD Symposium on Unsteady Aerodynamic-Fundamentals and Applications to Aircraft Dynamics, AGARD CP 386 USA (1985), Pp. 25-1 to 25-15.

[11] TOBAK (M.), ÜNAL (A.). Bifurcations in unsteady aerodynamics. NASA TM-88316 USA (June 1986).

[12] VOLTERRA (V.). Theory of functionals and of integral and integrodifferential equations. Dover Pub. Inc., New York (1959).

[13] COLEMAN (B. D.), NOLL (W.). An approximation theorem for functionals with applications in continuum mechanics. Arch. Rational Mech. Anal., 6, USA (1960), pp. 355-370.

[14] NOWINSKI (J. L.). Note on the applications of the Fréchet derivative. Int. J. Non-Linear Mech., 18, No. 4, USA (1983), pp. 297-306.

[15] BROOKE BENJAMIN (T.). Bifurcation phenomena in steady flows of a viscous fluid. I, Theory. II, Experiments. Proc. Roy. Soc. London, Ser. A, 359; USA (1978), pp. 1-43.

[16] JOSEPH (D. D.). Stability of fluid motions I. Springer-Verlag, New York $(1976)$.

[17] JOSEPH (D. D.). Hydrodynamic stability and bifurcation. Hydrodynamic Instabilities and the Transition to Turbulence: Topics in Applied PhysIcs, 45, H. L. Swinney and J. P. Gollub, eds., Springer-Verlag, New York USA (1981), pp. 27-76.

[18] SATTINGER (D. H.). Bifurcation and symmetry breaking in applied mathematics. Bull. (New Series) Amer. Math. Soc., 3, No. 2, USA (1980), pp. 779-819. 
[19] LANFORD (O. E.). Strange attractors and turbulence. Hydrodynamic Instabilities and the Transition of Turbulence: Topics in Applied Physics, 45, H. L. Swinney and J. P. Gollub, eds., Springer-Verlag, New York USA (1981), pp. 7-26.

[20] LADYZHENSKAYA (0. A.). The mathematical theory of viscous incompressible flow. Gordon and Breach, New York USA (1969).

[21] Iooss (G.). Bifurcation and transition to turbulence in hydrodynamics, Bifurcation Theory and Applications: Lecture Notes in Mathematics 1057 , L. Salvadori, ed., Springer-Verlag, New York USA (1984), pp. 152-201.

[22] GROSCH (C. E.), SALWEN (H.). The continuous spectrum of the OrrSommerfeld equation, Part 1: The Spectrum and the Eigenfunctions. $\underline{J}$. Fluid Mech., 87, Part 1, USA (1978), pp. 33-54,

[23] HERRON (I. M.). A diffusion equation illustrating spectral theory for boundary layer stability. Stud. Appl. Math., 67, No, 3, USA (Dec. 1982), pp. $231-241$.

[24] BOUARD (R.), COUTANCEAU (M.). The early stage of development of the wake behind an impulsively started cylinder for $40<\operatorname{Re}<10^{4}$, J. Fluid Mech. 101, Part 3, USA (1980), pp. 583-607.

[25] LOC (T. P.), BOUARD (R.). Numerical solution of the early stage of the development of the unsteady viscous flow around a circular cylinder: a comparison with experimental visualization and measurement. J. Fluid Mech., 160, USA (1985), pp. 93-117.

[26] NISHIOKA (M.), SATO (H.), Mechanism of determination of the shedding frequency of vortices behind a cylinder at low Reynolds numbers. J. Fluid Mech., 89, Part 1, USA (1978), pp. 49-60.

[27] KEENER (E, R.). Flow separation patterns on symmetric forebodies. NASA TM-86016, USA (Jan. 1986). 
[28] PEAKE (D. J.), TOBAK (M.). Three-dimensional interactions and vortical flows with emphasis on high speeds. AGARDograph No. 252 (1980). Also NASA TM-81169, USA (March 1980).

[29] TOBAK (M.), PEAKE (D. J.). Topology of three-dimensional separated flows. Ann. Rev. Fluid Mech., 14, USA (1982), pp. 61-85.

[30] CHAPMAN (G. T.). Topological classification of flow separation on threedimensional bodies. AIAA Paper 86-4085, USA (Jan. 1986).

[31] MORKOVIN (M. V.). On the question of instabilities upstream of cylindrical bodies. NASA CR-3231, USA (1979).

[32] BROOKE BENJAMIN (T.). Theory of vortex breakdown phenomenon. J. Fluid Mech., 4, Part 4, USA (1962), pp. 593-629.

[33] HWANG (C.), PI (W. S.). Investigation of steady and fluctuating pressures associated with the transonic buffeting and wing rock of a oneseventh scale model of the F-5A aircraft. NASA CR-3061, USA (1978).

[34] SARPKAYA (T.). Vortex-induced oscillations. J. Appl. Mech., 46, No. 2 (June 1979), pp. 241-258.

[35] BEARMAN (P. W.). Vortex shedding from oscillating bluff bodies. Ann. Rev. Fluid Mech., 16, USA (1984), pp. 195-222.

[36] FARMER (J. D.), OTT (E.), YORKE (J. A.). The dimension of chaotic attractors. Physica, 7D, USA (1983), pp. 153-180. 
Fig. 1 Maneuver referred to space-fixed $(X, Y)$ and moving $(x, y)$ coordinates.

Maneuvre se rapportant aux coordinées fixées dans l'éspace $(X, Y)$ et aux coordinées en mouvement $(x, y)$.

Fig. 2 Formation of indicial response.

Formation de la réponse indicial.

Eig. 3 Passage of angle of attack through a critical value $a_{c}$.

Passage de l'angle de l'attaque au travers d'une valeur critique $a_{c}$.

Fig. 4 Body and coordinates for impulsive-flow analogy.

Corps et coordinées pour l'analogie de l'écoulement impulsif.

Fig. 5 Folds in solution curve for rolling-moment coefficient $c_{\ell}$.

Plis dans la courbe des solutions pour le coéfficient du moment de roulis $C_{\ell}$. 
Fig. 6 Lines of separation on a body of revolution at angle of attack.

(a) $\alpha=15^{\circ} ;$ (b) $\alpha=35^{\circ}$.

Lignes de séparation sur un corps de révolution incliné à une angle de l'attaque. (a) $\alpha=15^{\circ} ;$ (b) $\alpha=35^{\circ}$.

Eig. 7 Major subdivisions of aerodynamic force.

Subdivisions principales de la force aerodynamique.

Fig. 8 Memory effects

Effets de la mémoire.

Fig. 9 Flexibly mounted cylinder immersed in a uniform oncoming stream.

(a) Spring-mass system; (b) Bifurcation diagram for stationary cylinder immersed in a uniform oncoming stream.

Cylindre suspendu sur des ressorts et immersé dans un écoulement uniform. (a) Système ressort-mass; (b) Diagramme de bifurcation pour un cylindre stationnaire immersé dans un écoulement uniform. 


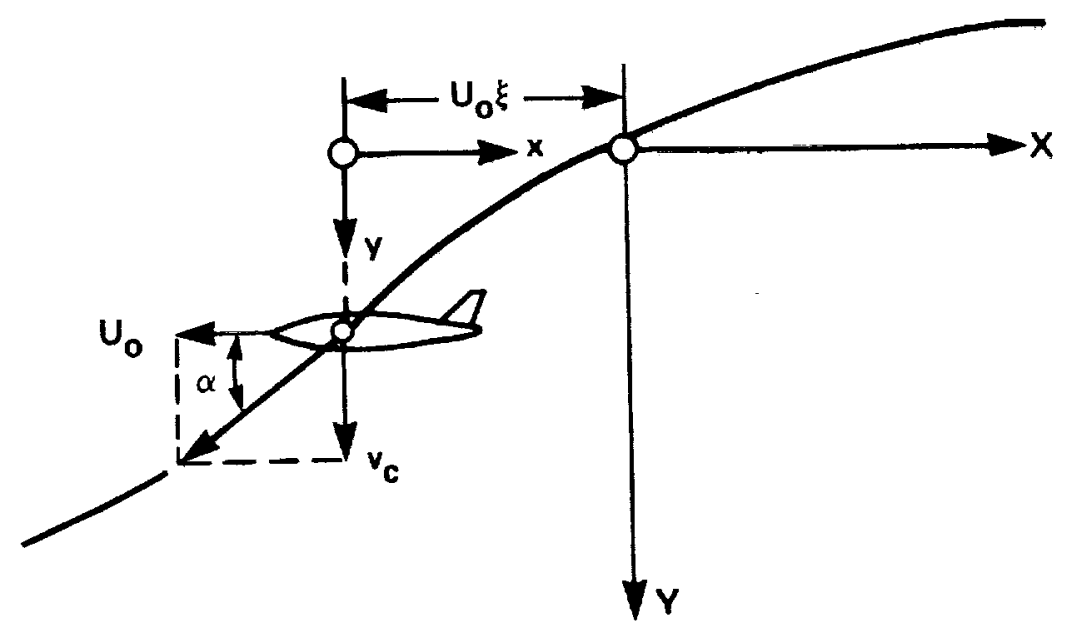

F1g. 1
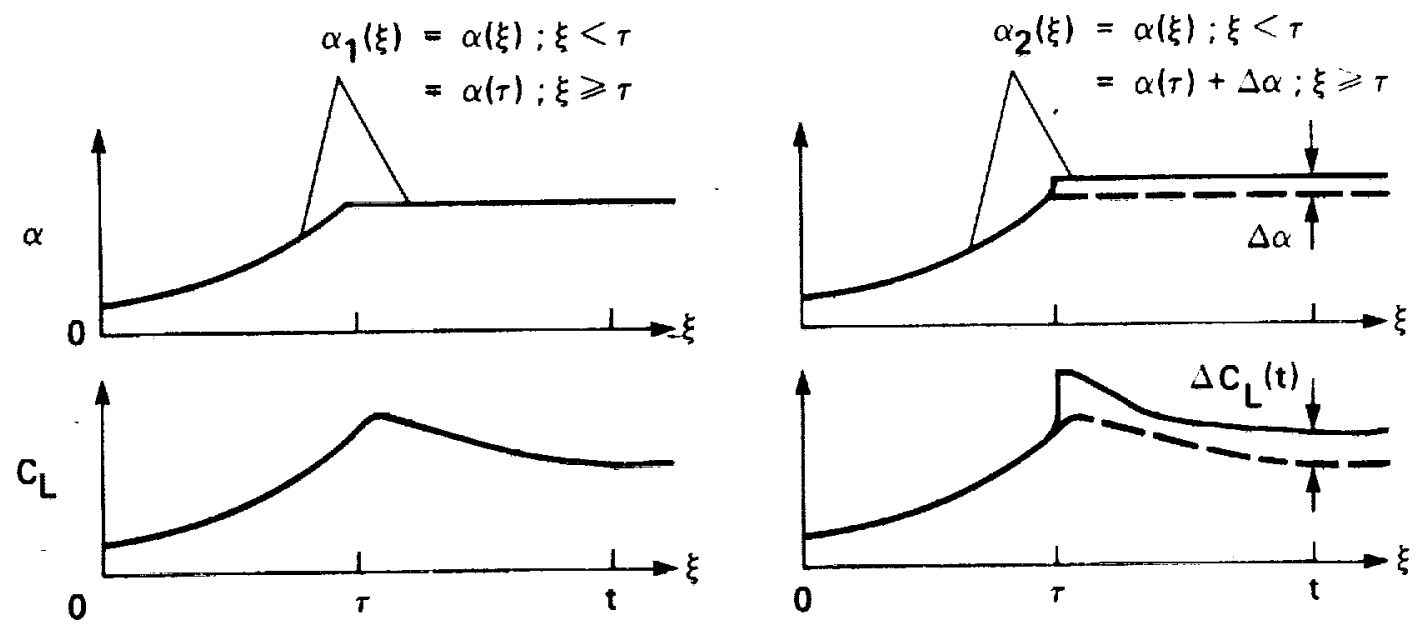

F1g. 2

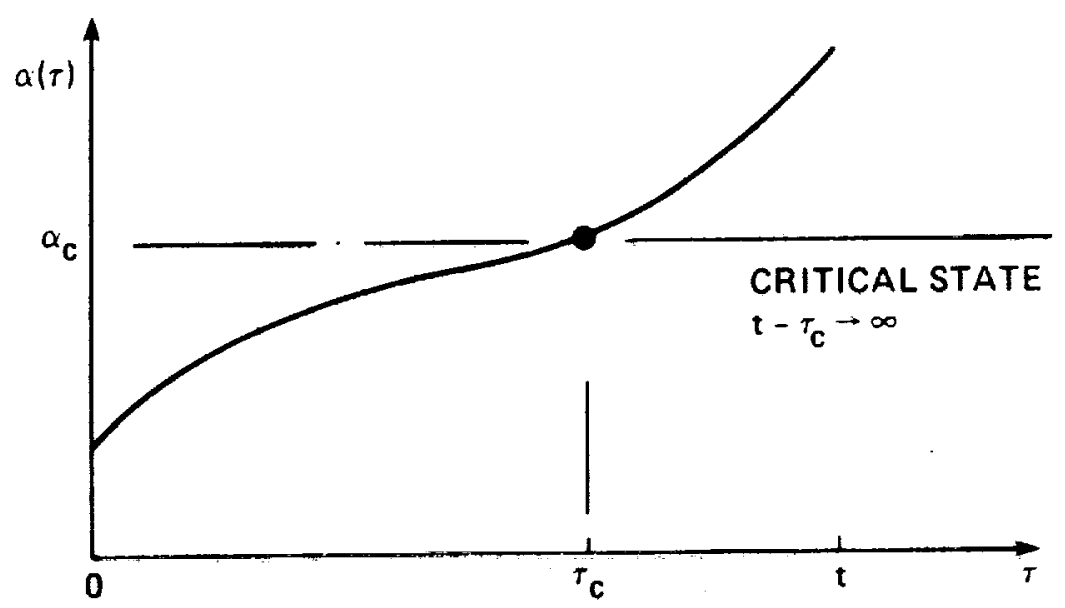

F1g. 3 


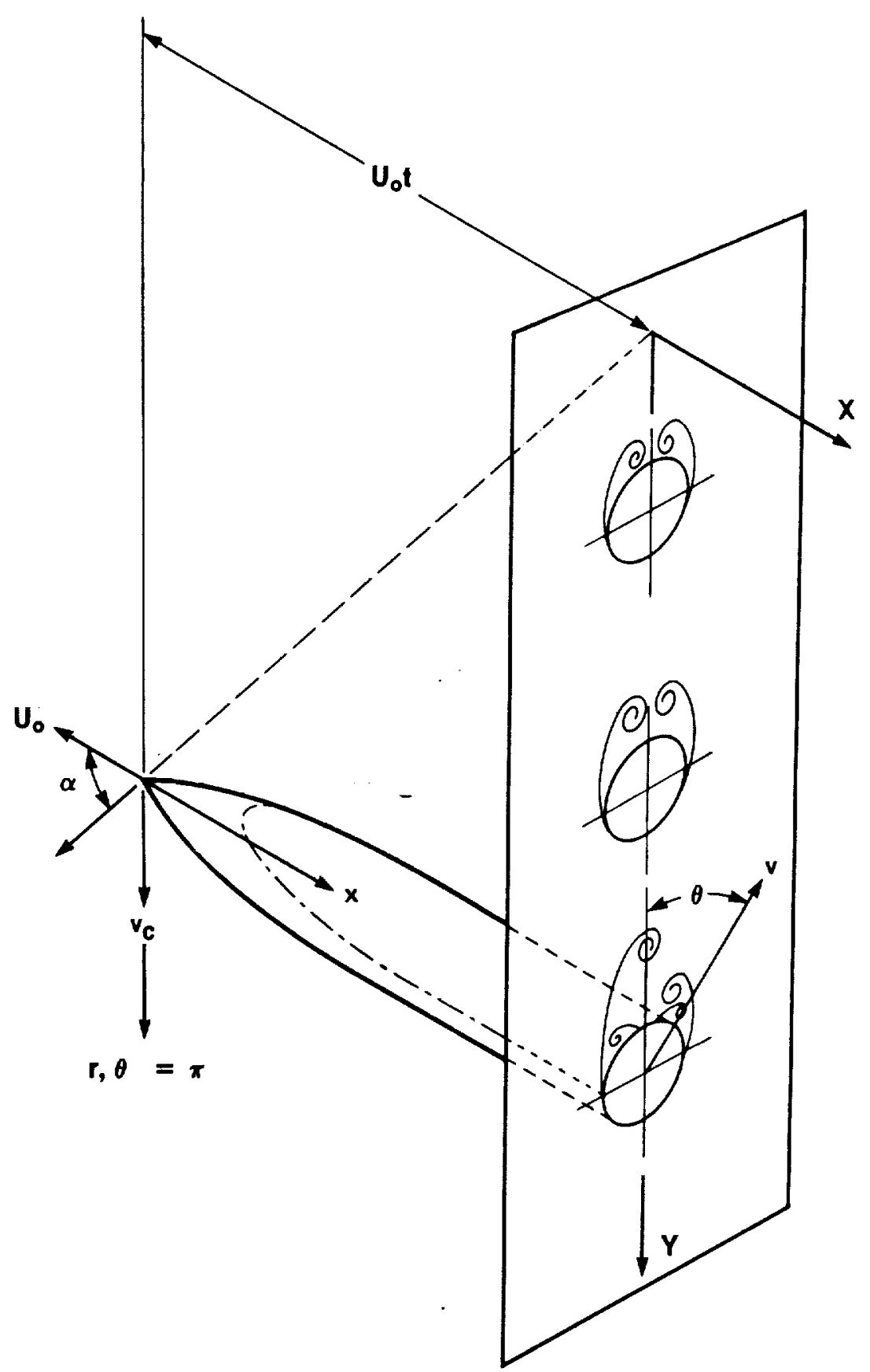

Fig. 4 


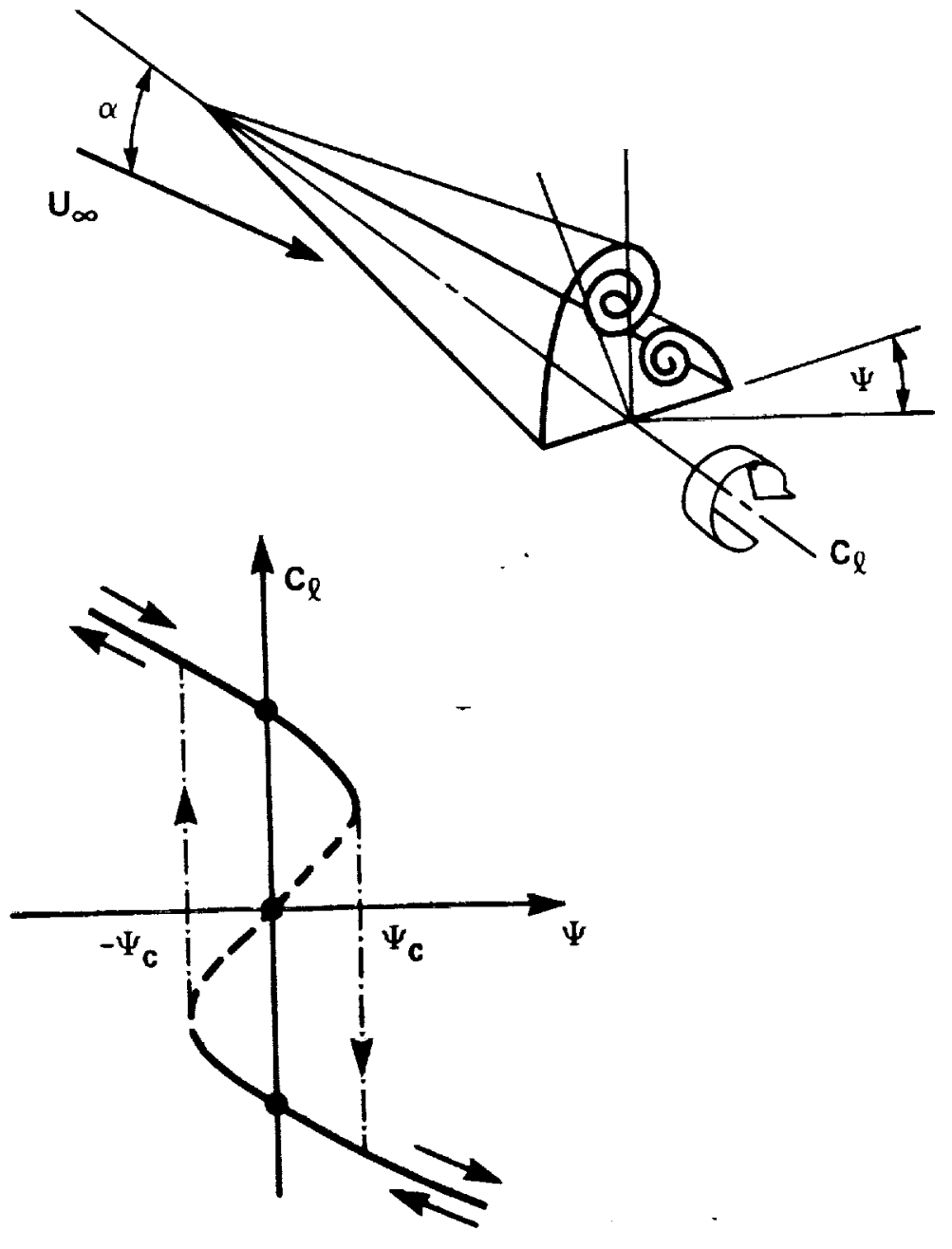

Fig. 5 
ORIGINAE PAGE IS

OF POOR QUALIT'

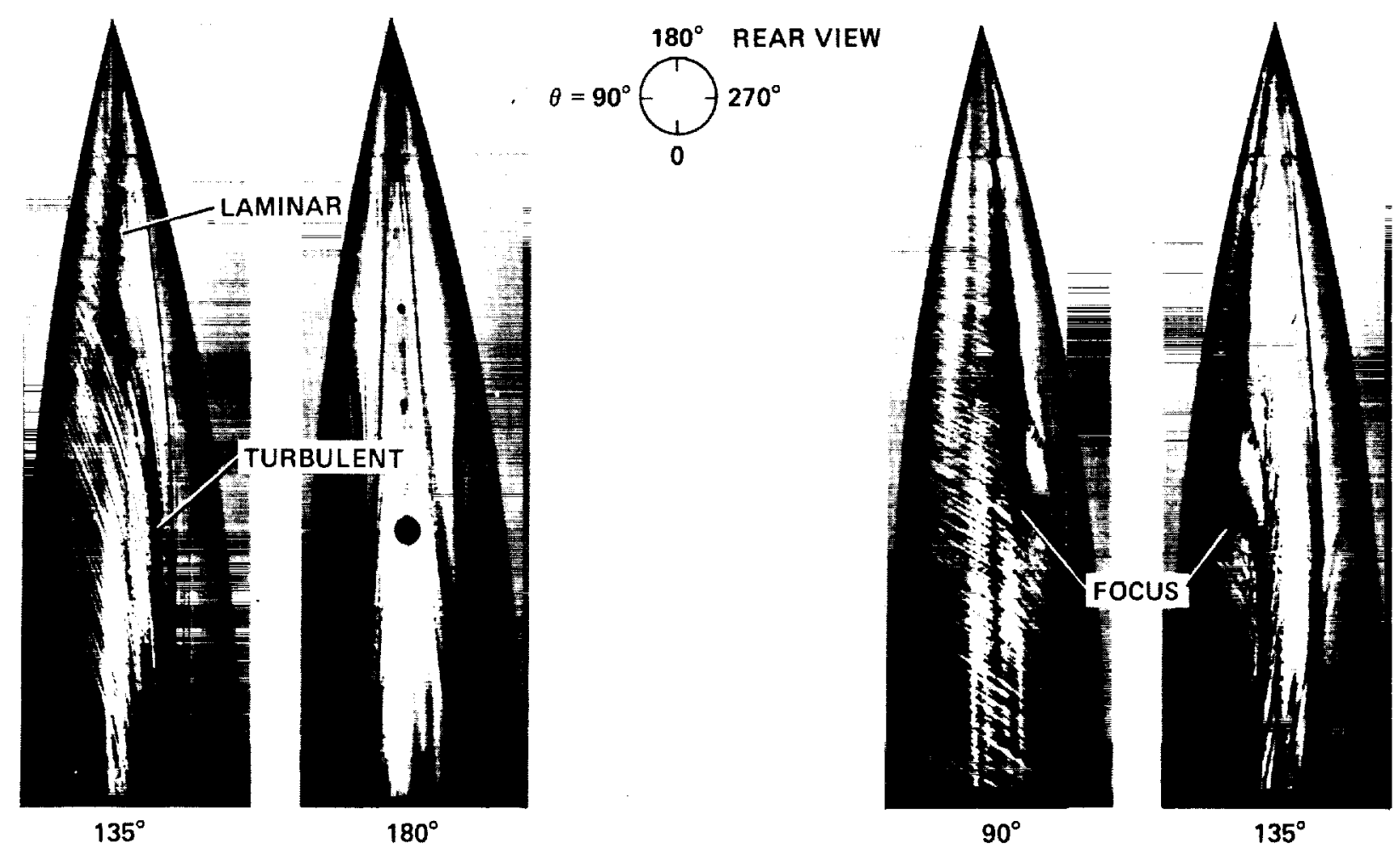

(a) $\alpha=15^{\circ}$

(b) $\alpha=35^{\circ}$

Fig. 6
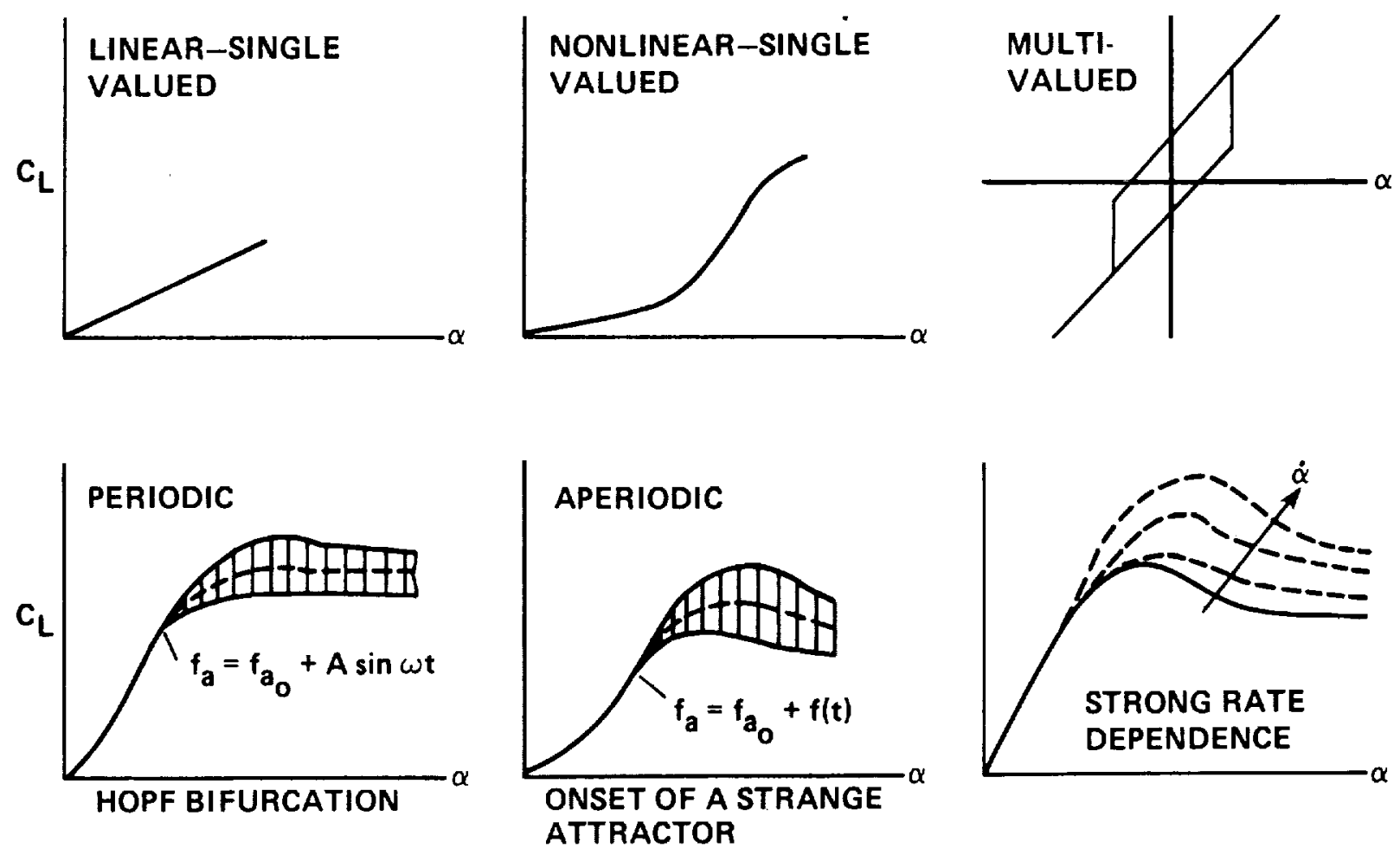

Fig. 7 

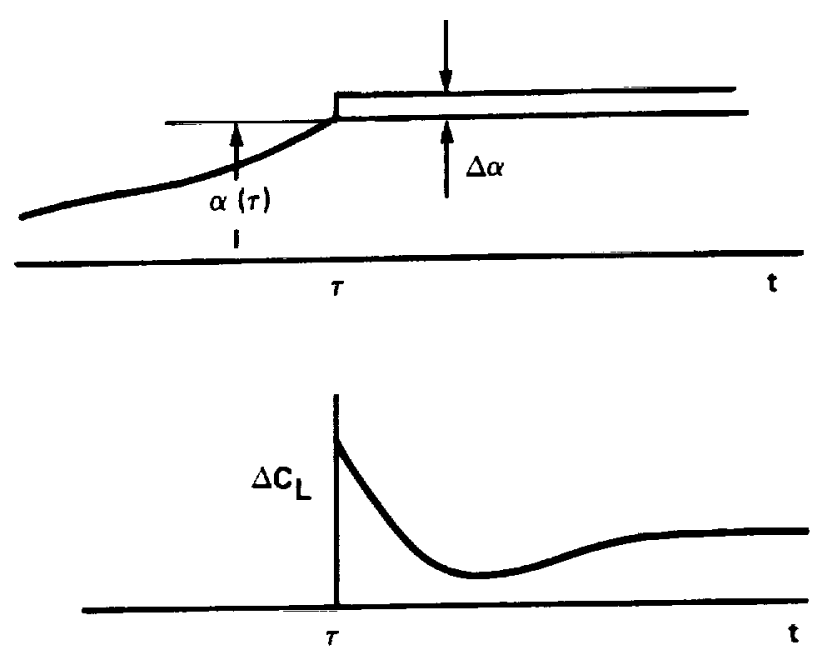

(a) TIME-INVARIANT EQUILIBRIUM STATE
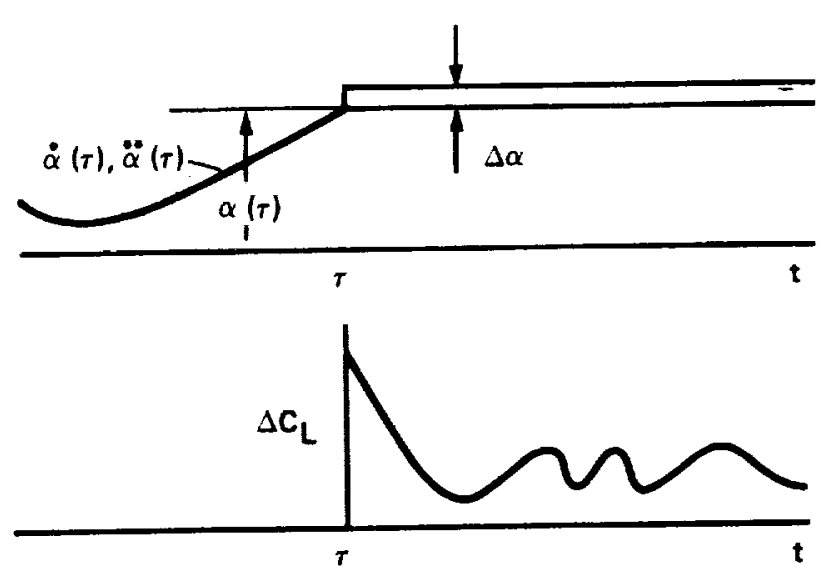

(c) QUASIPERIODIC EQUILIBRIUM STATE
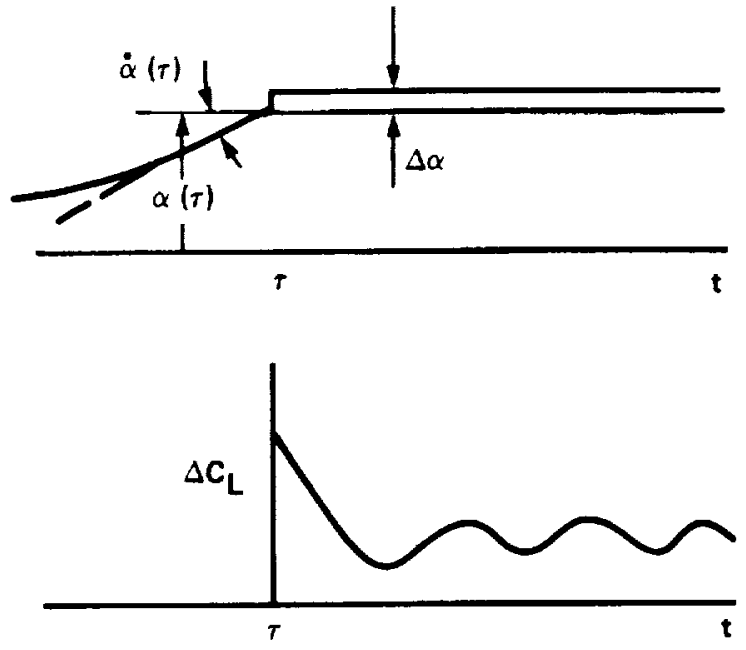

(b) PERIODIC EQUILIBRIUM STATE
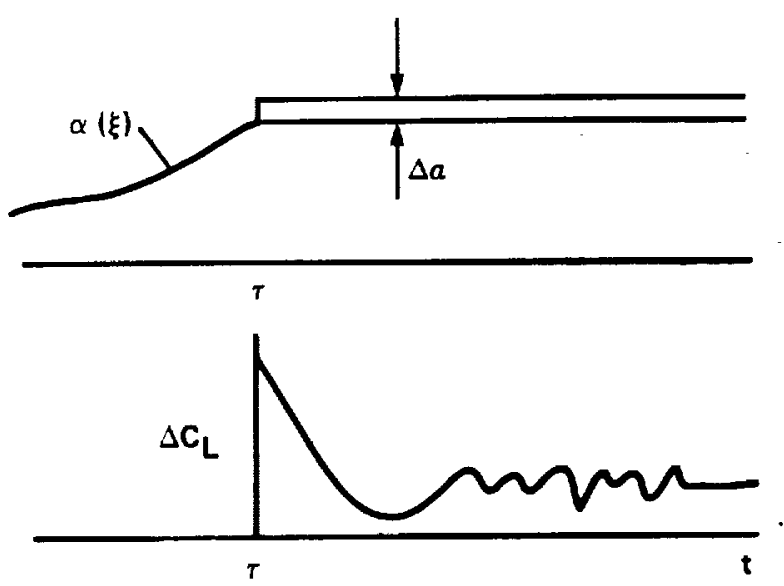

(d) CHAOTIC EQUILIBRIUM STATE

Fig. 8 


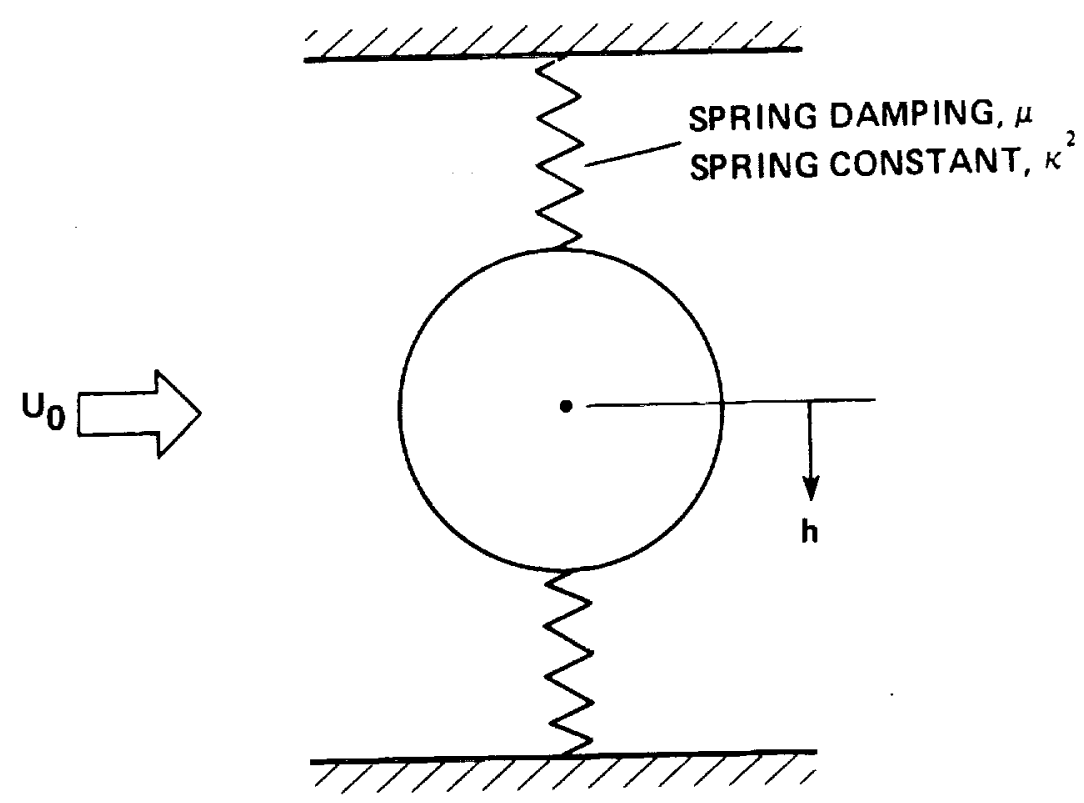

(a)

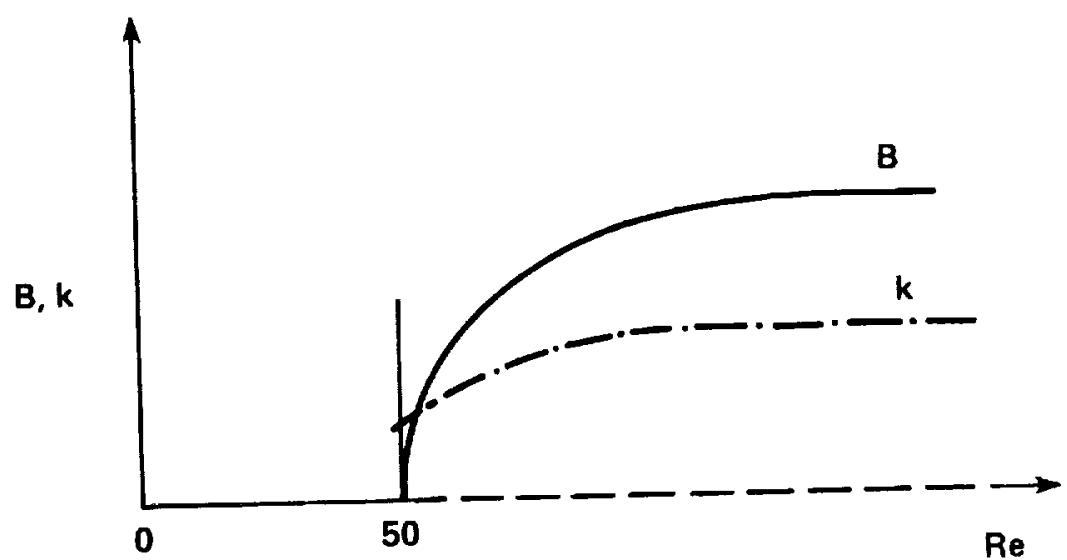

(b)

Fig. 9 


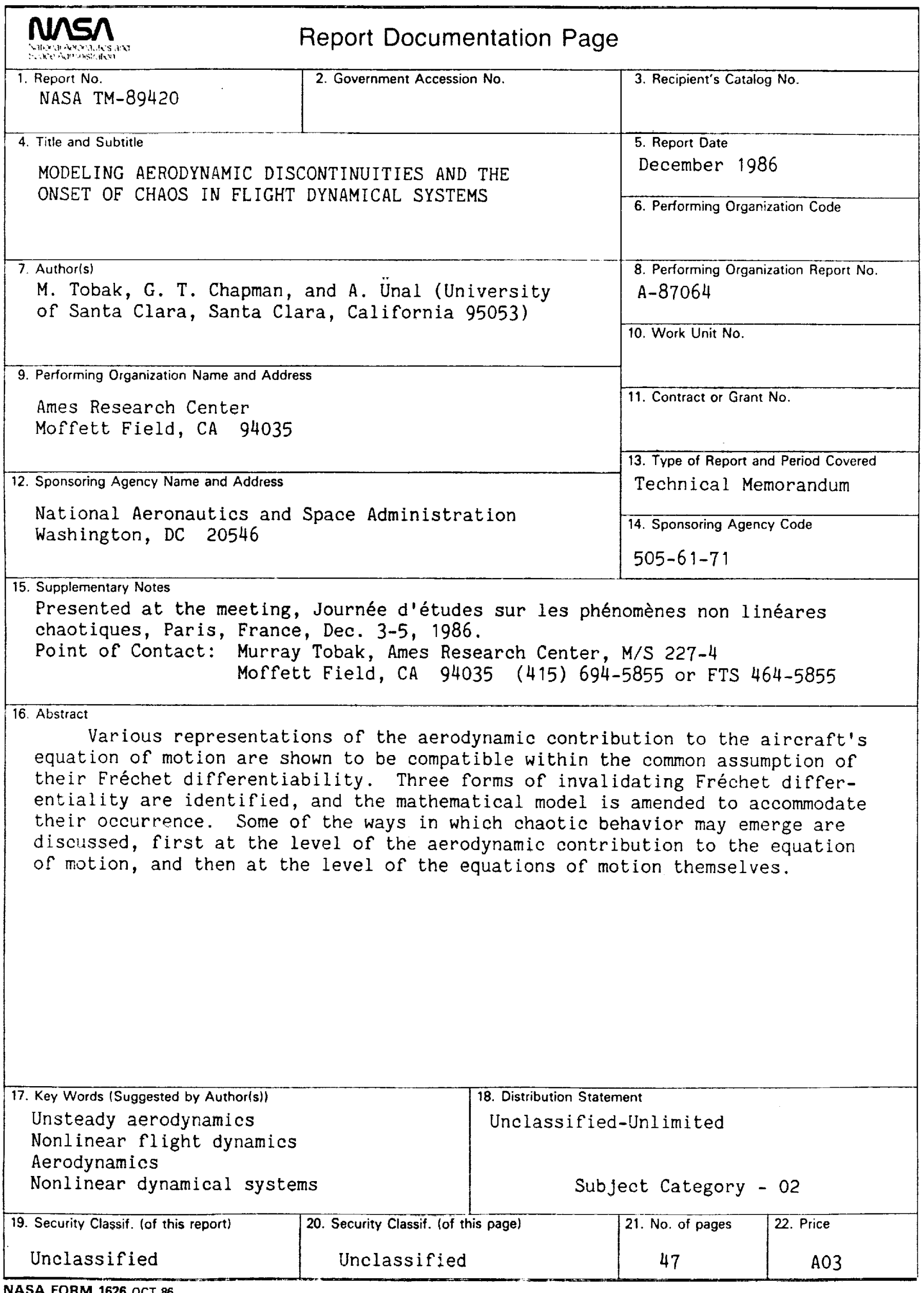

